\title{
Microscope-less Lab-on-a-chip Raman Spectroscopy of Cell-membranes
}

\author{
Ashim Dhakal $^{1,2}$, Pieter Wuytens ${ }^{1,2,3}$, Frédéric Peyskens ${ }^{1,2}$, Andre Skirtach ${ }^{2,3}$, \\ Nicolas Le Thomas ${ }^{1,2}$ and Roel Baets ${ }^{1,2}$ \\ ${ }^{1}$ Photonics Research Group, INTEC Department-Ghent University-imec, \\ ${ }^{2}$ Center for Nano- and Biophotonics, Ghent University, B-9000 Ghent, Belgium \\ ${ }^{3}$ Department of Molecular Biotechnology, Ghent University, B-9000 Ghent, Belgium \\ ashim.dhakal@ugent.be
}

\begin{abstract}
We investigate the membranes of cells fixed on a photonic chip using evanescent excitation and collection of Raman signals using single-mode nano-photonic waveguides. Enhancement and selectivity of the evanescent interaction allow for selectively probing the membrane with higher efficiency than confocal microscopes.
\end{abstract}

Keywords-Raman spectroscopy, Cell Membranes, Integrated photonics, Lab-on-a-chip

\section{INTRODUCTION}

The cell membrane is a complex arrangement of phospholipids, proteins and carbohydrates. These membranebound molecules regulate most of the vital biological activities of the cells by controlling the transmembrane and intracellular transport. Due to the lack of sensitive, selective and in-situ tools, the study of these membrane-bound molecules is largely done in isolation from the cells or from the membranes. Damaging methods of enrichment such as centrifugation are used to enhance the signal. The fact that about $60 \%$ of all drugs target the activity of native membrane molecules underscores the importance of having a selective Raman tool for the study of cell membranes in its natural state [1]. Here we report the possibility of using a lab-on-a-chip method to obtain selective Raman spectra from the cell-membranes of the cells grown directly on a chip.

Raman spectroscopy of cell-membranes is based on the use of the fundamental TE mode of single mode silicon nitride $\left(\mathrm{Si}_{3} \mathrm{~N}_{4}\right)$ on silica waveguides fabricated using CMOScompatible process steps. Single mode photonic waveguides have previously been used to implement Raman spectroscopy of bulk liquids in a lab-on-a-chip framework [2, 3]. It has been shown that a large interaction area and high field enhancement near the vicinity of high index contrast waveguides reduce the signal-to-noise ratio (SNR) or the signal acquisition time by at least two orders of magnitude compared to the traditional method using a confocal microscope [4]. In addition, the étendue of the collected signal in a single-mode waveguide is the least, which allows smallest possible integrated spectrometers with an optimal spectral resolution. A low cost and high performance Raman sensors for the study of the cellmembranes can thus be integrated on a chip.

\section{EXPERIMENT AND METHODS}

First we note that the $\mathrm{SiO}_{2}$ and $\mathrm{Si}_{3} \mathrm{~N}_{4}$ materials used for our waveguides are not adverse to the overall cell growth and are

routinely used as biocompatible materials for the cell culture [5]. Further, as shown in Fig. 1 and Fig. 2, the cells adhere to the waveguide and the membrane following its topography, such that the evanescent tail of the waveguide mode interacts with the membrane, allowing one to probe exclusively the region very close to the waveguides. For $850 \mathrm{~nm}$ wide slotted waveguides (inclusive of a $150 \mathrm{~nm}$ slot region) we use the collected Raman signal halves every $20 \mathrm{~nm}$ away from the waveguide surface.

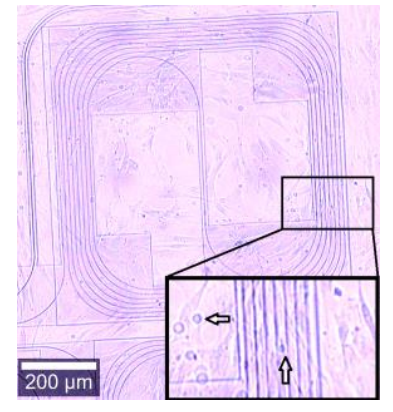

Fig. 1. Microscope image of the HeLa cells grown on top of a $\mathrm{Si}_{3} \mathrm{~N}_{4}$ waveguide spiral carrying the pump laser as well as the Raman signal. The rectangular selection in the base zooms in a section of the top of the spiral where the cells and their organelles (shown with arrows) can be seen.
HeLa cells (ATCC) were cultured in Advanced Dulbecco's Modifed Eagle's Medium (GIBCO-LT12491-015) supplemented with $2 \%$ fetal bovine serum

(GIBCO-LT-10500-056) and $1 \%$ penicillinstreptomycin-L-glutamine

(GIBCO-LT-10378-016), and grown in culture flasks containing a $\mathrm{Si}_{3} \mathrm{~N}_{4}$ chip and a $\mathrm{CaF}$ slide in an incubator at $37{ }^{\circ} \mathrm{C}$ and $5 \% \mathrm{CO}_{2} . \mathrm{CaF}$ slide was used to obtain the spectra with a confocal microscope for comparison. Prior to fixation, cells were washed in the phosphate buffered saline (PBS). Fixation on the $\mathrm{Si}_{3} \mathrm{~N}_{4}$ chip and $\mathrm{CaF}$ slide was performed for 10 minutes in $4 \%$ paraformaldehyde solution, after which cells were washed three times and stored in PBS.

The setup used to measure the Raman signal from the membranes collected by the waveguides has been explained elsewhere [2]. A 785 $\mathrm{nm}$ pump with $30 \mathrm{~mW}$ power is coupled to the waveguide with about $8 \pm 2 \mathrm{~dB}$ coupling loss per facet. The copropagating light is collected, filtered and coupled to a single mode fiber to measure the Raman spectrum with a commercial spectrometer

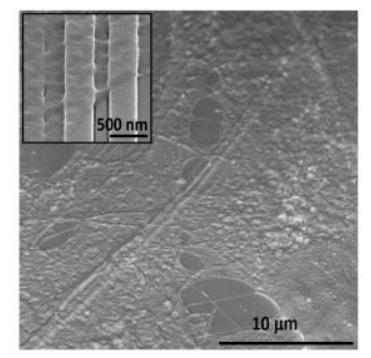

Fig. 2. A SEM micrograph of a cell fixed to the surface of the $\mathrm{Si}_{3} \mathrm{~N}_{4}-\mathrm{SiO}_{2}$ chip. A good adhesion of the cells with the surface of the chip is observed. 
(Andor SR303i) and a $-80{ }^{\circ} \mathrm{C}$ cooled $\mathrm{CCD}$ detector (Andor iDUS 416).

Fig. 3 illustrates the background-subtracted Raman spectra of HeLa cells measured using a commercial confocal microscope (WITec Alpha300R+ confocal Raman microscope) by specifically focusing at the cell membrane using a z-scan, with a confocal height of $1.5 \mu \mathrm{m}$. This limited z-resolution inhibits a selective probing of the cell membrane, a problem which is resolved using our on-chip approach. The figure also shows two background-subtracted example spectra of the cells grown on the chip and measured by evanescently exciting and collecting the membrane molecules using the waveguide. The typical integration times were less than $10 \mathrm{~s}$ for an effective pump power of $500 \mu \mathrm{W}$ in the waveguide to obtain a signal-tonoise ratio $(\mathrm{SNR})>50$. To obtain a spectrum with a similar SNR using similar pump power, the integration time needed for a confocal microscope would be $>500 \mathrm{~s}$.

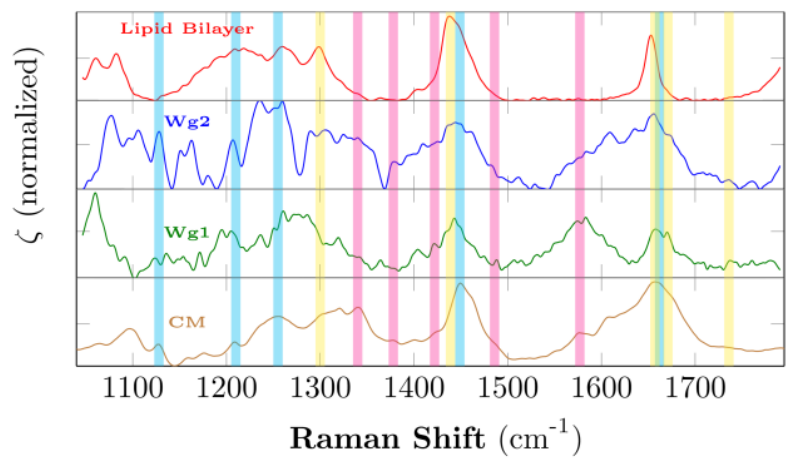

Fig.3. Raman spectra obtained from commercial confocal microscope (CM) of the cells grown on Raman grade CaF slides, NERS spectra from the cells grown on top of the chips containing the waveguides (Wg1, Wg2) and the spectra from the lipid bilayer formed on top of the waveguide. Light cyan, yellow and pink lines are due to nucleic acids, lipid and proteins respectively [7]. The typical integration times were less than 10 seconds for an effective pump power of $500 \mu \mathrm{W}$ in the waveguide.

To simulate cell membranes consisting of only lipids, the spectrum of a lipid bilayer formed on the chip using the vesicle [6] (using vesicles formed by 4:4:2 mixture of dioleoyl phosphatidylethanolamine, dioleoyl phosphatidyl choline, and Cholesterol) and evanescently measured using the waveguides is displayed. In contrast to the spectrum of the lipid bilayer, the spectra of the cells additionally contain distinctive peaks [7] due to proteins and nucleic acids. The spectra measured using the waveguide have some similarity with the one obtained using the confocal microscope, but several distinctive peaks due to proteins and nucleic acids become more prominent with the waveguide due to exclusivity and localization of the Raman interaction near the membranes.

Interestingly, the spectra of the cells differ from one waveguide to another while retaining certain general characteristics, such as the peaks related to lipid. A possible reason for this difference between the spectra of the membranes can be the complex and heterogeneous nature of the cell membrane that alters significantly depending on the stress conditions of the individual cells in their respective microenvironments. Other reasons could be the presence of specific cell debris on the waveguide, or the location of specific parts of the cell in the slotted region where the evanescent interaction is maximal. A more detailed study is needed to ascertain the origin of the various peaks and to explain the spectral differences. Nevertheless, our study suggests that the on-chip method is suitable to the study of cell membranes in their native state and can bring down the integration times to the order of sub-seconds (with SNR >10 instead of $>50$ ) leading to a pathway for real-time analysis.

\section{CONCLUSIONS}

In conclusion, evanescently excited and collected Raman spectra provide several distinctive features that can be assigned to proteins and nucleic acids. These peaks are not as distinct in the spectra obtained with a confocal microscope. We ascribe these features of the Raman spectra collected using the waveguides to the exclusivity and localization of the evanescent Raman interaction near the membrane-waveguide contact region. Further, the increased interaction area with the optical mode results in an enhanced signal compared to a commercial confocal microscope. The enhanced signal results in a signal acquisition time below $10 \mathrm{~s}$ for a SNR > 50. Our preliminary study suggests that the on-chip method using the nanophotonic waveguides is suitable for studying cell membranes in their native state, while also bringing down the integration times to the order of sub-seconds with a reasonable SNR. This leads to a pathway for a real-time analysis of the interactions that happen in the cell-membranes.

\section{ACKNOWLEDGMENT}

The authors acknowledge the ERC advanced grant InSpectra, Fonds Wetenschappelijk Onderzoek (FWO) and Bijzonder Onderzoeksfonds (BOF-UGent) for financial support, and imec for fabrication of the waveguides. We also thank Jia $\mathrm{Su}$, Kherim Willems and Susana Rocha from KULeuven-imec for providing the lipid vesicles.

\section{REFERENCES}

[1] M. A. Yildirim, K. I. Goh, M. E. Cusick, A. L.Barabasi, M. Vidal, "Drug-target Network", Nat Biotechnol, Vol 25 (10), 2007, p. 1119-26.

[2] A. Dhakal, A.Z. Subramanian, P. Wuytens, F. Peyskens, N. Le Thomas, R. Baets, "Evanescent excitation and collection of spontaneous Raman spectra using silicon nitride nanophotonic waveguides", Opt. Lett., Vol 39 (13), 2014, p. 4025-4028.

[3] A. Dhakal, A. Raza, F. Peyskens,A.Z. Subramanian, S. Clemman, N. Le Thomas, R. Baets," Efficiency of evanescent excitation and collection of spontaneous Raman scattering near high index contrast channel waveguides", Opt. Express, Vol 23 (21), 2015, p.27391-27404

[4] A. Dhakal, A. Raza, P.C. Wuytens, F. Peyskens, A. Skirtach, R. Baets, "Lab-on-a-chip Raman sensors outperforming Raman microscopes, accepted for publication" in CLEO 2016, USA

[5] E.A. Carter, B.S.Rayner, A.I. McLeod, L.E.Wu, C.P. Marshall, et. al.,. "Silicon nitride as a versatile growth substrate for microspectroscopic imaging and mapping of individual cells", Mol. Biosystems, Vol 6 (7), 2010, p .1316-1322.

[6] R. P. Richter, R. Bérat. and A. R. Brisson, "Formation of solidsupported lipid bilayers: an integrated view", Langmuir, Vol 22(8), 2006, p. 3497-3505.

[7] C. Krafft, T. Knetschke, A.Siegner, R. H. Funk and R. Salzer, "Mapping of single cells by near infrared Raman microspectroscopy", Vibr. Spectr., Vol 32(1), 2003, p. 75-83 